\title{
Video Article \\ Examining the Role of Nasopharyngeal-associated Lymphoreticular Tissue (NALT) in Mouse Responses to Vaccines
}

\author{
Emily D. Cisney ${ }^{1}$, Stefan Fernandez ${ }^{1}$, Shannan I. Hall ${ }^{1}$, Gale A. Krietz ${ }^{1}$, Robert G. Ulrich ${ }^{1}$ \\ ${ }^{1}$ U.S. Army Medical Research Institute of Infectious Diseases
}

Correspondence to: Robert G. Ulrich at rulrich@bhsai.org

URL: https://www.jove.com/video/3960

DOI: doi:10.3791/3960

Keywords: Infectious Diseases, Issue 66, Immunology, nasal vaccination, nasopharyngeal-associated lymphoreticular tissue, mouse, antibody, mucosal immunity, NALT ablation, NALT culture, NALT-deficient mice

Date Published: 8/1/2012

Citation: Cisney, E.D., Fernandez, S., Hall, S.I., Krietz, G.A., Ulrich, R.G. Examining the Role of Nasopharyngeal-associated Lymphoreticular Tissue (NALT) in Mouse Responses to Vaccines. J. Vis. Exp. (66), e3960, doi:10.3791/3960 (2012).

\section{Abstract}

The nasopharyngeal-associated lymphoreticular tissues (NALT) found in humans, rodents, and other mammals, contribute to immunity in the nasal sinuses ${ }^{1-3}$. The NALT are two parallel bell-shaped structures located in the nasal passages above the hard palate, and are usually considered to be secondary components of the mucosal-associated lymphoid system ${ }^{4-6}$. Located within the NALT are discrete compartments of $B$ and $T$ lymphocytes interspersed with antigen-presenting dendritic cells ${ }^{4,7,8}$. These cells are surrounded by an epithelial cell layer intercalated with M-cells that are responsible for antigen retrieval from the mucosal surfaces of the air passages ${ }^{9,10}$. Naive lymphocytes circulating through the NALT are poised to respond to first encounters with respiratory pathogens ${ }^{7}$. While NALT disappear in humans by the age of two years, the Waldeyer's Ring and similarly structured lymphatic organs continue to persist throughout life ${ }^{6}$. In contrast to humans, mice retain NALT throughout life, thus providing a convenient animal model for the study of immune responses originating within the nasal sinuses ${ }^{11}$.

Cultures of single-cell suspensions of NALT are not practical due to low yields of mononuclear cells. However, NALT biology can be examined by ex vivo culturing of the intact organ, and this method has the additional advantage of maintaining the natural tissue structure. For in vivo studies, genetic knockout models presenting defects limited to NALT are not currently available due to a poor understanding of the developmental pathway. For example, while lymphotoxin- $\alpha$ knockout mice have atrophied NALT, the Peyer's patches, peripheral lymph nodes, follicular dendritic cells and other lymphoid tissues are also altered in these genetically manipulated mice ${ }^{12,13}$. As an alternative to gene knockout mice, surgical ablation permanently eliminates NALT from the nasal passage without affecting other tissues. The resulting mouse model has been used to establish relationships between NALT and immune responses to vaccines ${ }^{1,3}$. Serial collection of serum, saliva, nasal washes and vaginal secretions is necessary for establishing the basis of host responses to vaccination, while immune responses originating directly from NALT can be confirmed by tissue culture. The following procedures outline the surgeries, tissue culture and sample collection necessary to examine local and systemic humoral immune responses to intranasal (IN) vaccination.

\section{Video Link}

The video component of this article can be found at https://www.jove.com/video/3960/

\section{NALT Collection and Culturing}

1. Euthanize mice using approved IACUC guidance. Avoid use of inhalant anesthetics that may affect NALT. Transfer mice to an aseptic workspace or biosafety cabinet. Remove the lower jaw of the mouse and clean the upper palate area with alcohol and iodine wipes.

2. Use a No. 11 surgical blade in surgical knife handle to carefully cut and excise the upper palate by following the inside contour of the mouse incisors and molar teeth.

3. Gently peel back the palate with forceps, being careful not to tear the palate.

4. This step is necessary to reduce contamination of cultures and is designed for processing multiple palates. Place palates into individual wells in the first column of a sterile 48 -well plate pre-filled with $250 \mu \mathrm{L}$ of complete culture medium $\left(37^{\circ} \mathrm{C}\right)$ consisting of RPMI 1640 supplemented with $10 \%$ fetal bovine serum, $100 \mu \mathrm{g} / \mathrm{mL}$ streptomycin, $100 \mathrm{Ul} / \mathrm{mL}$ penicillin, $50 \mu \mathrm{g} / \mathrm{mL}$ gentamicin and $1 \mu \mathrm{g} / \mathrm{mL}$ fungizone/amphotericin. Media should be carbonate buffered if palates are cultured in a $5 \% \mathrm{CO}_{2} / 95 \%$ humid air incubator $\left(37^{\circ} \mathrm{C}\right)$, or alternatively use $10 \mathrm{mM} \mathrm{HEPES}$ $\mathrm{pH} 7.4$, for non- $\mathrm{CO}_{2}$ culture.

5. To wash the palates, use forceps to move palates into each successive well in a row, carefully tapping the plate between washes, until the palate has undergone a total of eight washes.

6. Transfer the palates into a new sterile 48 -well plate containing $250 \mu \mathrm{L}$ of fresh, complete culture medium $\left(37^{\circ} \mathrm{C}\right)$ in wells.

7. Place the plates containing the palates into a $37^{\circ} \mathrm{C}$ incubator for culturing for the duration of the study.

8. Collect samples for analysis by aseptically transferring $100 \mu \mathrm{L}$ of medium from culture wells into Eppendorf tubes every 24 hours, replacing with $100 \mu \mathrm{L}$ of fresh $37^{\circ} \mathrm{C}$ culture medium. 
9. Centrifuge the culture media samples at $380 \mathrm{xg}$ for 10 minutes, $4^{\circ} \mathrm{C}$, to pellet debris.

10. Transfer the supernatant from the centrifuged sample to fresh Eppendorf tubes and store at $-20^{\circ} \mathrm{C}$ until ready to analyze.

11. Antigen-specific $\operatorname{lgG}, \lg \mathrm{M}$, and IgA, or secreted cytokines are measured in tissue-culture supernatants by standard enzyme-linked immunosorbent assays (ELISA).

\section{Surgical Ablation of NALT}

1. Female BALB/c mice, 7 to 9 weeks of age, are suitable subjects, but other strains are also acceptable. Provide gel-like wet food three days prior to surgery to acclimate mouse to food and water substitute that will be administered post-operation.

2. Administer one drop of Metacam pain relief medication $(1.5 \mathrm{mg} / \mathrm{ml}$ or $0.05 \mathrm{mg} / \mathrm{drop})$ into the oral cavity prior to surgery.

3. Anesthetize mice using approved IACUC guidance, for example, with a ketamine, acepromazine, and xylazine mixture (KAX) and apply Puralube Vet Ointment onto eyes to prevent drying. Avoid use of inhalant anesthetics that may affect NALT. Anesthesia can be confirmed by the absence of reflex response to a gentle pinching of the toes.

4. Administer $1 \mathrm{~mL}$ saline (0.9\% sodium chloride injection USP) subcutaneously between shoulder blades using a 22-28 gauge needle and syringe to prevent potential dehydration of the mouse after surgery. Also administer $0.1 \mathrm{~mL}$ of Respiram subcutaneously between shoulder blades using a 22-28 gauge needle and syringe to promote healthy respiration during and after surgery.

5. Provide thermal support to the mouse during all subsequent manipulations.

6. Place the anesthetized mouse in a supine position, and use two separate loops of surgical suture around the upper and lower incisors to gently pry open the mouth, exposing the upper palate.

7. Use a No. 11 surgical blade to make an incision approximately $3 \mathrm{~mm}$ in length down the midline of the upper palate in the site of the NALT.

8. Insert a $0.5 \mathrm{~mm}$ microcurette into the incision and gently scrape under the edges to disrupt the NALT.

9. Cauterize the incision to stop bleeding with a straight fine loop on a thermal cautery unit.

10. Continue thermal support while mouse recovers to full consciousness and activity.

11. Provide mouse with saline subcutaneously up to three times a day, $1 \mathrm{~mL}$ per injection between shoulder blades, to prevent dehydration as necessary and oral pain medication once a day for 3 days post surgery. Determine dehydration by performing a skin tent test to check for skin turgor. Grasp the skin and fur between the shoulders blades so that it is tented up. If the skin quickly returns to the normal, previous position, the mouse is not dehydrated. If the skin remains elevated and moves slowly, the mouse is in a state of dehydration and requires saline. Provide wet food for at least three days after surgery to allow sufficient time for recovery.

12. Prior to any experimental procedures, observe mice for 8-10 days by tracking weight gain. Poor weight recovery usually indicates incomplete palate healing, and these mice should be withdrawn from further studies.

\section{Preparing NALT for Histology and Assessing the Success of NALT Surgery}

1. The success of NALT surgery must be ascertained by histology as described below. After completing all experimental studies, prepare mice for cranial collections by euthanizing using approved IACUC guidance. Avoid use of inhalant anesthetics that may affect NALT.

2. Grasp the nape of the neck of the euthanized mouse. Remove the lower mandible from the rest of the skull by snipping through the condylar processes with scissors on both sides.

3. Starting at the nape of the neck, remove the skin and fur from the skull by slowly peeling and cutting towards the ventral side of the cranium

4. Carefully remove skin around the nasal area and snip the skin off of the tip of the snout to remove completely.

5. Detach the cranium from the vertebrae with a snip of the scissors.

6. Insert scissors into the foramen magnum and cut halfway down the cranium along the midline to allow permeation of formalin into the soft tissues during fixation.

7. Fix cranium in $10 \%$ neutral buffered formalin (NBF) for 24 hours at room temperature.

8. To decalcify, place the sample and separate cassette, wrapped in gauze to prevent Rexyn from touching the bone, in bottle with the formic acid mixture. Incubate for 12 hours at room temperature, then rinse continuously under tap water for approximately 20 minutes.

9. Trim the sample to the septum and past the eye orbits with a razor blade.

10. Sample and cassette can be stored for short-term in $10 \%$ NBF.

11. Place sample onto a tissue processor for overnight processing. This is done to remove water from the sample in preparation for paraffin embedding.

12. Remove processed tissue, embed in a paraffin block with the snout down, and allow it to cool.

13. Trim rough $15 \mu \mathrm{m}$ cross-sections of the block with an automated microtome, approaching the approximate location of the NALT, then continue cutting in $5 \mu \mathrm{m}$ sections for mounting onto glass slides in a $44.3^{\circ} \mathrm{C}$ water bath.

14. Hematoxylin and eosin (H\&E) histology staining should be used to assess NALT ablation.

\section{Collection of Biological Samples from Mice}

\subsection{Serum}

1. Carefully elevating body temperature with a heat lamp will increase blood flow. Collect blood by nicking the lateral tail vein with a surgical blade. Allow the blood to drip freely into a Microtainer serum separator tube.

2. Apply gentle pressure to nick with absorbent material, such as gauze or towel, to stop blood flow.

3. Allow the blood to clot in the Microtainers for 30 minutes, then centrifuge between $6-15 \times 1,000 \mathrm{~g}$ for at least 90 seconds.

4. Transfer separated serum from Microtainer into a clean Eppendorf tube for storage at $-80^{\circ} \mathrm{C}$.

\subsection{Saliva}

1. Hold the anesthetized mouse vertically while pipetting 20-30 $\mu \mathrm{L}$ sterile phosphate buffered saline (PBS) between the cheek and gum line. Collect the diluted saliva by directing pipette tip between cheek and gum line. 
2. Transfer the diluted saliva to a fresh tube containing $10 \mu \mathrm{L}$ of $2 \mathrm{x}$ protease inhibitor and store at $-20^{\circ} \mathrm{C}$.

\subsection{Nasal secretions}

1. Euthanize mouse before collecting nasal secretions, using approved IACUC guidance. Avoid use of inhalant anesthetics that may affect NALT.

2. Holding the mouse vertically, carefully pipette $30 \mu \mathrm{L}$ of sterile PBS in one nostril and collect rinse from other nostril.

3. Transfer rinse to a fresh tube containing $10 \mu \mathrm{L}$ of $2 x$ protease inhibitor and store at $-20^{\circ} \mathrm{C}$.

\subsection{Vaginal secretions}

1. Insert the tip of a micropipette into the opening of the vulva of a euthanized mouse, rinse the vagina with $50 \mu \mathrm{L}$ of sterile PBS and collect all fluid by micropipette.

2. Transfer rinse to a fresh tube containing $10 \mu \mathrm{L}$ of $2 x$ protease inhibitor and store at $-20^{\circ} \mathrm{C}$.

\section{Antigen-specific antibodies or cytokines in the collected samples can be measured by ELISA or another quantitative method.}

\section{Representative Results}

Figure 1 provides a general schematic of steps involved with processing the NALT-containing tissue for ex vivo analysis. In Figure 2 (A, B), the size of the palate is shown, as well as the location of the incision during ablation surgery (A), as indicated by the dotted line. The location of NALT are indicated by arrows in the premolar area on an excised hematoxylin-stained palate in Figure 2 (C), showing the parallel tissues.

Figure 3 presents steps of the NALT disruption surgery, showing exposure of the upper palate for access to NALT (A, B), ablation (C), and final cauterization of the incision (D). A typical $\mathrm{H}$ \& $\mathrm{E}$ cross-section of the nasal sinus area surrounding the NALT before surgery is shown in Figure $3 \mathrm{E}$, while an image of NALT disruption by the microcurette directly after surgery appears in Figure 3F. Allowing sufficient time for recuperation from surgery, the incisions should be closed and the nasal cavity devoid of NALT (Figure 3G).

Typical experimental results obtained by using these techniques are shown in Figure 4, comparing tissue culture supernatants and biological samples from a study of a staphylococcal subunit vaccine (STEBVax). Mice were administered STEBVax by intranasal (IN) or intraperitoneal (IP) routes. The vaccine was formulated with an adjuvant that activates the Toll-like receptor 4 pathway, ${ }^{3,14}$, and controls were given only saline or vaccine without adjuvant. Cultured NALT obtained from experimental groups secreted antigen-specific immunoglobulins into medium that was measurable by ELISA. In this example (Figure 4A), the results indicate that the greatest amounts of IgA were released by NALT obtained from mice vaccinated IN with a subunit vaccine combined with adjuvant.

Biological samples (such as serum, saliva, nasal secretions, vaginal secretions) acquired from control or NALT-free mice can be used to profile the in vivo immune response to nasal antigens for comparison with the tissue culture results. In Figure 4B, IgA and IgG responses to IN vaccination were significantly decreased without functional NALT. Levels of antigen-specific IgA were generally greater than IgG in mucosal secretions (saliva, nasal washes) of vaccinated mice. 




Figure 1. Schematic of NALT collection and ex vivo culturing.



Figure 2. Visualization of the mouse palate indicating the position of NALT and surgery incision. Size and location of upper palate with surgery incision denoted by dotted line (A); upper palate excised (B) or stained in hematoxylin (C) to view the parallel NALT in the premolar area of palate (stained dark purple on anterior side of palate). NALT indicated by arrows. 

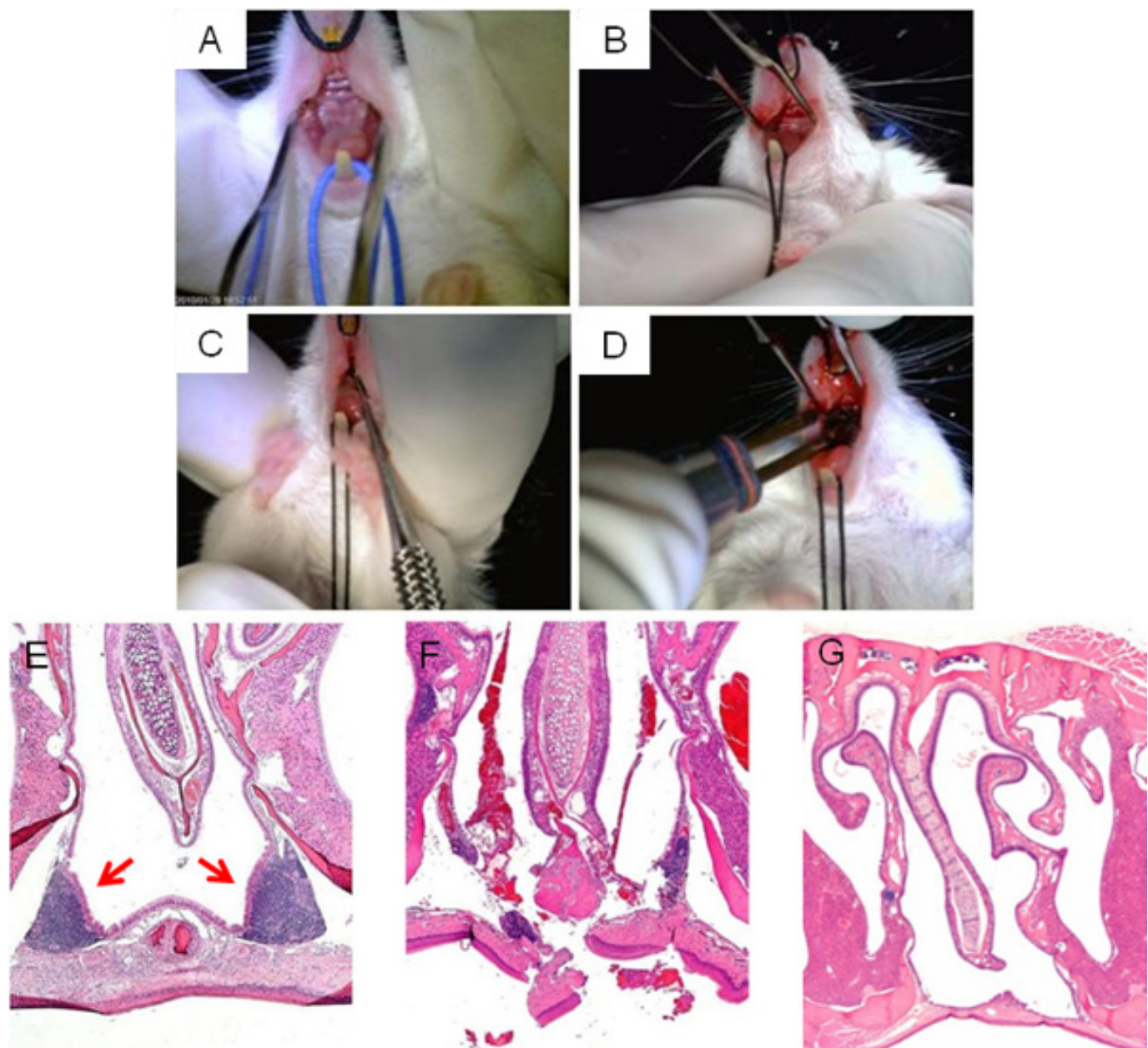

Figure 3. Surgical NALT disruption. Key stages of NALT disruption surgery: supine view of mouse upper palate before surgery (A); midline incision made on upper palate to access the NALT (B); microcurette probe inserted through midline incision to disrupt NALT structure integrity (C); cauterization of incision at conclusion of surgery (D). Microscopic images of nasal cavities, H\&E stained, before surgery (E); immediately after surgery (F); and a successfully healed, NALT-free mouse (G). 

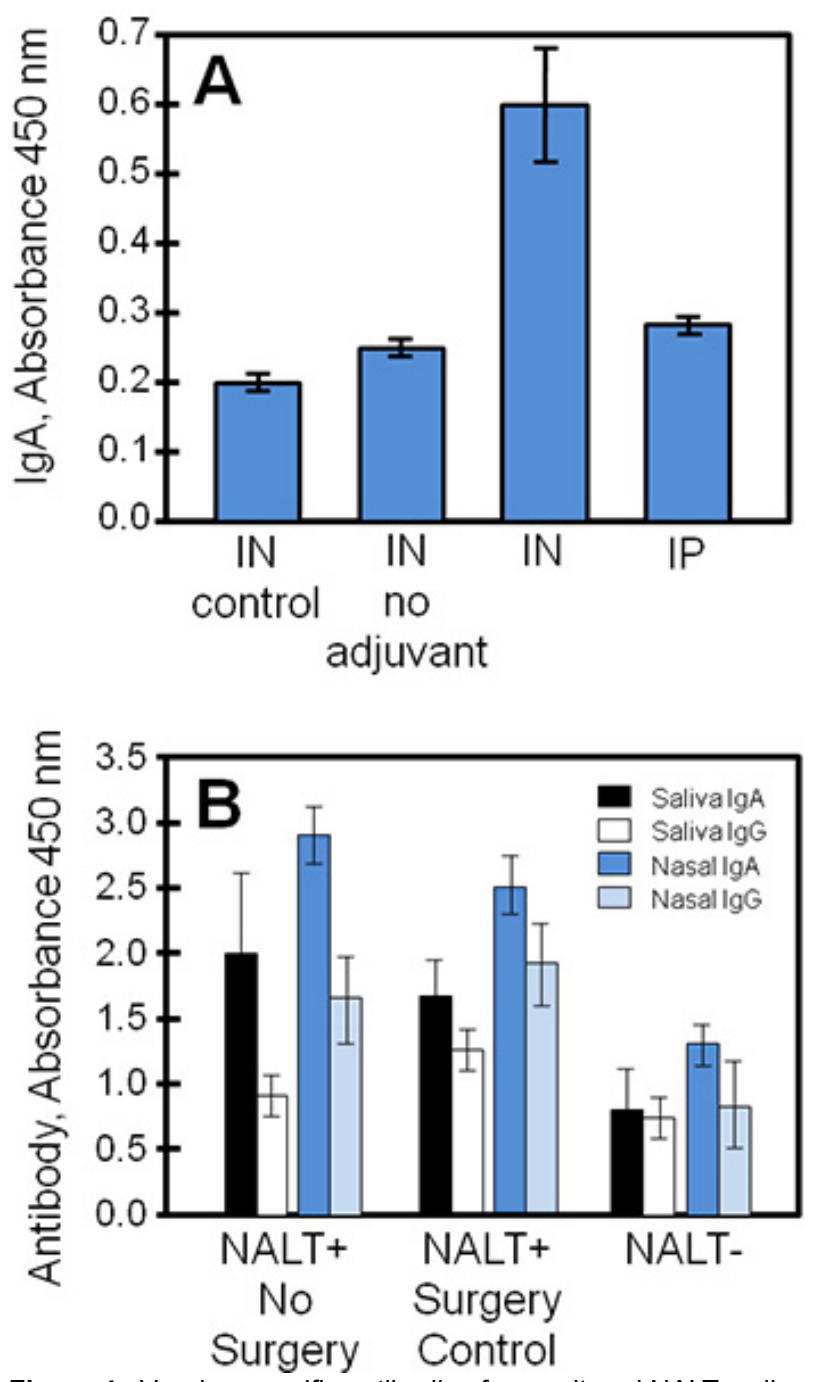

Figure 4. Vaccine-specific antibodies from cultured NALT, saliva, and nasal secretions collected from vaccinated mice. NALT-free or normal control mice were vaccinated IN or IP with STEBVax, and biological samples were collected. Antibody levels of triplicate samples were measured using ELISA. (A) NALT were removed from control mice (not surgically manipulated) and cultured to examine antibody responses. The vaccinespecific IgA response in culture for IN vaccinated mice was statistically different from controls (Student's t-test, $p \leq 0.01$, compared to no adjuvant or no vaccine). (B) NALT disruption substantially reduced specific antibody responses to IN vaccination. There were significant differences between specific antibody levels of NALT- and NALT+ groups (no surgery or control surgery) for all comparisons except saliva IgG results (Student's t-test, $\mathrm{p} \leq 0.05$ ).

\section{Discussion}

We have presented collective methods for developing an animal model, obtaining biological samples, and assays for examining NALTassociated immune responses ${ }^{1-4}$. There are additional factors to consider during the performance of these methods. Standard sterile techniques for surgery and tissue culture should be followed. A combination of antibacterial and antifungal agents used during isolation and culture, as well as maintaining sterilized instruments, work area, and disinfected palates will reduce the risk of contamination. Saliva, nasal washes and similar mucosal secretion samples should be inspected for potential contamination with blood, as serum antibodies are generally found in higher concentrations. Further, mucosal secretions should only be slightly diluted for analysis because lower concentrations of antibodies are present in these samples compared to serum.

Mice must be kept warm directly after surgery to prevent potential anesthesia-induced hypothermia. Alternate resting mice on their sides during post-surgery recuperation to minimize irregular respiration. The surgical procedure is more efficient with three individuals working together to complete these tasks: one performing the surgery, one to assist in holding the mouth open, and one to provide post-surgical care as the mice recover from anesthesia.

It is necessary to use H\&E staining of cranial cross-sections at the end of all experimental procedures or studies to verify the success of the surgery for each mouse. Possible surgery outcomes are: complete and bilateral NALT ablation, incomplete ablation, or intact NALT. Because not all surgeries will result in complete loss of the NALT, animals with residual or intact NALT can be used as internal controls. Another potential 
outcome is that the palate fails to completely heal, leaving an opening connecting the nasal and the oral cavities. Incomplete healing of the palate will result in low weight and failure to thrive, and these individuals should be removed from studies.

Examining vaccine responses first with the mouse model will serve to establish a role for NALT in the intended study outcome (antibody response, survival, etc.). Surgically removing the NALT facilitates the determination of nasal contributions to local and systemic immunity. The surgical approach described here is the most direct method for obtaining a mouse model devoid of NALT. Select knock-out mouse models have been reported to lack NALT, but these animals also are deficient in cytokines or chemokines essential to the development of other secondary lymphoid tissues, and may harbor additional defects ${ }^{12,13}$. Further, the methods described here were developed for examining several aspects of immune responses originating within the nasal passages. Our experimental results are based on studies using the entire upper palate from the mouse for tissue culture, although it is possible that sections may be used. Finally, the cultured NALT model is useful for performing experiments completely in tissue culture.

\section{Disclosures}

No conflicts of interest declared.

\section{Acknowledgements}

Support was provided by Becton Dickinson Technologies. Views expressed in this submission are those of the authors and do not purport to reflect official policy of the U.S. Government. Research was conducted in compliance with the Animal Welfare Act and other federal statutes and regulations relating to animals and experiments involving animals and adheres to principles stated in the Guide for the Care and Use of Laboratory Animals, National Research Council, 1996. The facility where this research was conducted is fully accredited by the Association for Assessment and Accreditation of Laboratory Animal Care International.

\section{References}

1. Wiley, J.A., Tighe, M.P., \& Harmsen, A.G. Upper respiratory tract resistance to influenza infection is not prevented by the absence of either nasal-associated lymphoid tissue or cervical lymph nodes. J. Immunol. 175, 3186-3196 (2005).

2. Zuercher. A.W., et al. Intranasal immunisation with conjugate vaccine protects mice from systemic and respiratory tract infection with Pseudomonas aeruginosa. Vaccine. 24, 4333-4342 (2006)

3. Fernandez, S., Cisney, E.D., Hall, S.I., \& Ulrich, R.G. Nasal immunity to staphylococcal toxic shock is controlled by the nasopharynxassociated lymphoid tissue. Clin. Vaccine Immunol. 18, 667-675 (2011).

4. Asanuma, H., et al. Isolation and characterization of mouse nasal-associated lymphoid tissue. J. Immunol. Methods. 202, 123-131 (1997).

5. Casteleyn, C., Broos, A.M., Simoens, P., \& Van den Broeck, W. NALT (nasal cavity-associated lymphoid tissue) in the rabbit. Vet. Immunol. Immunopathol. 133, 212-218 (2010).

6. Debertin, A.S., et al. Nasal-associated lymphoid tissue (NALT): frequency and localization in young children. Clin. Exp. Immunol. 134, 503-507 (2003).

7. Csencsits, K.L., Jutila, M.A., \& Pascual, D.W. Nasal-associated lymphoid tissue: phenotypic and functional evidence for the primary role of peripheral node addressin in naïve lymphocyte adhesion to high endothelial venules in a mucosal site. J. Immunol. 163, 1382-1389 (1999).

8. Zuercher, A.W., et al. Nasal-associated lymphoid tissue is a mucosal inductive site for virus-specific humoral and cellular immune responses. J. Immunol. 168, 1796-1803 (2002).

9. Park, H.S., Francis, K.P., Yu, J., \& Cleary, P.P. Membranous cells in nasal-associated lymphoid tissue: a portal of entry for the respiratory mucosal pathogen group A streptococcus. J. Immunol. 171, 2532-2537 (2003).

10. Tyrer. P., Foxwell, A.R., Cripps, A.W., Apicella, M.A., \& Kyd, J.M. Microbial pattern recognition receptors mediate M-cell uptake of a gramnegative bacterium. Infect. Immun. 74, 625-631 (2006).

11. Wu, A.W. \& Russell, M.W. Nasal lymphoid tissue, intranasal immunization, and compartmentalization of the common mucosal immune system. Immunol. Res. 16, 187-201 (1997).

12. Harmsen, A., et al. Cutting edge: organogenesis of nasal-associated lymphoid tissue (NALT) occurs independently of lymphotoxin-alpha (LT alpha) and retinoic acid receptor-related orphan receptor-gamma, but the organization of NALT is LT alpha dependent. J. Immunol. 168, 986-900 (2002).

13. Rangel-Moreno, J., et al. Role of CXC chemokine ligand 13, CC chemokine ligand (CCL) 19, and CCL21 in the organization and function of nasal-associated lymphoid tissue. J. Immunol. 175, 4904-4913 (2005).

14. Morefield, G.L., Hawkins, L.D., Ishizaka, S.T., Kissner, T.L., \& Ulrich, R.G. Synthetic Toll-like receptor 4 agonist enhances vaccine efficacy in an experimental model of toxic shock syndrome. Clin. Vaccine Immunol. 14, 1499-1504 (2007). 